\title{
Individualized drug screening based on next generation sequencing and patient derived xenograft model for pancreatic cancer with bone metastasis
}

\author{
ZHONGHAI GUAN ${ }^{1,2 *}$, HUANRONG LAN ${ }^{3 *}$, XIANGHENG CHEN ${ }^{4}$, \\ XIAOXIA JIANG ${ }^{2}$, XUANWEI WANG ${ }^{5}$ and KETAO JIN ${ }^{1}$
}

\begin{abstract}
${ }^{1}$ Department of Gastrointestinal Surgery, Shaoxing People's Hospital, Shaoxing Hospital of Zhejiang University, Shaoxing, Zhejiang 312000; ${ }^{2}$ Department of Surgical Oncology, The 1st Affiliated Hospital, College of Medicine, Zhejiang University, Hangzhou, Zhejiang 310003; ${ }^{3}$ Department of Breast and Thyroid Surgery, Shaoxing People's Hospital, Shaoxing Hospital of Zhejiang University, Shaoxing, Zhejiang 312000 ; ${ }^{4}$ Department of Minimally Invasive Surgery, The 2nd Xiangya Hospital of Central South University, Changsha, Hunan 421001; ${ }^{5}$ Department of Orthopedics, The 1st Affiliated Hospital, College of Medicine, Zhejiang University, Hangzhou, Zhejiang 310003, P.R. China
\end{abstract}

Received March 16, 2017; Accepted August 1, 2017

DOI: $10.3892 / \mathrm{mmr} .2017 .7213$

\begin{abstract}
The efficacy of traditional chemoradiotherapies for pancreatic cancer remains limited, and no effective targeted therapies or screening tests are currently available. Therefore more individualized drug screening is warranted for the clinical treatment of pancreatic cancer. A patient-derived xenograft (PDX) model of pancreatic cancer bone metastasis was established, and next-generation sequencing (NGS) was used to investigate the molecular characteristics of the cancer and screen for potential drugs. Immunohistochemical analysis was performed to validate that the PDX retained the molecular characteristics from the patient. Using NGS technology, 13 pancreatic-cancer-associated polymorphisms/mutations were identified out of 416 genes sequenced. Based on the sequencing results and associated literatures, AZD6244, a highly selective inhibitor against mitogen-activated protein kinase kinase $1(M E K 1)$, was chosen as a potential therapy. AZD6244, a highly selective MEK1 inhibitor, was evaluated as effective for the pancreatic cancer PDX model, and thus
\end{abstract}

Correspondence to: Dr Ketao Jin, Department of Gastrointestinal Surgery, Shaoxing People's Hospital, Shaoxing Hospital of Zhejiang University, 568 Zhongxing North Road, Shaoxing, Zhejiang 312000, P.R. China

E-mail: jinketao2001@zju.edu.cn

Dr Xuanwei Wang, Department of Orthopedics, The 1st Affiliated Hospital, College of Medicine, Zhejiang University, 79 Qingchun Road, Hangzhou, Zhejiang 310003, P.R. China

E-mail: drwangxuanwei@aliyun.com

${ }^{*}$ Contributed equally

Key words: next generation sequencing, patient-derived xenograft model, individualized drug screening, pancreatic cancer with bone metastasis may provide potential efficacy in the clinical treatment of the patient with pancreatic cancer investigated in the present study. The feasibility of the novel NGS-PDX based drug-screening pattern was demonstrated, and has a potential to improve individualized treatment for cancer.

\section{Introduction}

Pancreatic cancer is expected to be the second most lethal malignancy in the USA by 2020, and the 5-year survival rate for patients diagnosed with locally advanced or metastatic pancreatic cancer remains $<3 \%(1,2)$. The efficacy of traditional chemoradiotherapies for pancreatic cancer remains limited (3-5). However, no effective targeted therapies or screening tests for pancreatic cancer are recently available, and no clinically comfirmed biomarkers are available for identifying subsets of patients who might benefit from chemoradiotherapies or targeted theprapies (6-9). Different from frequent liver and peritoneum metastases, the bone metastasis rate of pancreatic cancers is quite low but reaches higher of about $7.3 \%$ with the improvement of the diagnosis and treatment level $(10,11)$. For pancreatic cancer patients, especially those in advanced or metastatic disease stages, individualized drug screening is urgently needed for the clinical treatment.

The lack of an appropriate in vivo model for preclinical studies has limited the mechanistic study of tumor resistance to anti-VEGF therapy. Patient-derived xenografts (PDXs), so-called Avatar models (12), have been increasingly widely used in various types of cancers for translational research in recent years, with the greatest advantage of its ability to better predict clinical tumor response (13). Accumulating evidence indicates that PDX is an reliable cancer research tool for drug screening and personalized medicine applications (14).

It is known that somatic genomic alterations alter the function of genes or pathways, thus resulting in tumorigenesis, metastasis, and resistance to therapies $(15,16)$. Therefore, precise molecular profiles of tumors will help to predict drug 
responses (17). Understanding the genomic landscape of CRC can contribute to drug screening (8,18-20). Large-scale sequencing projects has economically led to the rapid development and clinical popularization of next-generation sequencing (NGS) technologies (21). NGS can be a powerful tool to understand the genomic landscape of patients and mechanism of drug response, which thus might provide a more broad vision for clinically potential drug screening (22-24). Therefore, NGS technologies are being used by pharmaceutical companies throughout the drug discovery process (21).

In our previous studies, we established a series of PDX models of different tumor types and accumulated substantial experiences of drug evaluation, screening and mechanism exploration $(25,26)$. While in the present study, we established a PDX model by pancreatic cancer bone metastasis tumor tissues for evaluation of potential drugs for pancreatic cancer patient. In our study, in order to select the optimal therapy for the patient, the NGS technology was used for investigating of tumor molecular characteristics and searching for potential drugs, which were finally evaluated in the corresponding PDX model. The aim of our study is to demonstrate the feasibility of the novel NGS-PDX based drug screening pattern which has a great potential to improve the cancer individualized treatment.

\section{Materials and methods}

Reagents and drugs. AZD6244 (cat.no. S1008) and Capecitabine (cat. no. S1156) were purchased from Selleck Chemicals (Shanghai, China). The antibodies against ki-67, CK19, CK7, PCNA, Caspase-3, ERK, p-ERK, and $\beta$-actin were purchased from Abcam (Cambridge, UK).

Patient and tumor tissues. Pancreatic cancer bone metastasis (diagnosed as adenocarcinoma) tissues were obtained at surgery from a 67-year-old female patient. A single bone metastasis was imageologically found at the right pedicle of L2 vertebral arch, which means a high risk of fracture and paraplegia. In addition, the patient urged for operation treatment. The study was done in accordance with the principles of the Declaration of Helsinki and the International Conference on Harmonization and Good Clinical Practice guidelines. The Institutional Ethical Committee approved the current study.

Establishment of PDX model. BALB/c nude mice (3-to-4-week-old, female) were purchased from Shanghai Slaccas Laboratory Animal and housed in SPF laboratory animal rooms at laboratory animal center of Zhejiang University. Mice were acclimated to new environments for at least 3 days before use. Surgical tumor tissues were cut into pieces of 3 to $4 \mathrm{~mm}$ and transplanted within $30 \mathrm{~min}$ s.c. to mice. Additional tissues were snap-frozen and stored at $-80^{\circ} \mathrm{C}$ until use. Animals were monitored periodically for their weight with an electronic balance and tumor growth with a Vernier caliper twice every week. The tumor volume was calculated as formula $\mathrm{V}=\mathrm{LD} \times(\mathrm{SD})^{2} / 2$, where $\mathrm{V}$ represents the tumor volume, LD and SD are the longest and the shortest tumor diameter, respectively. Tumors were then harvested, minced and re-implanted as described above for passaging. At each generation, tumors were harvested and stored in liquid nitrogen for further use. The usage of experimental animals
Table I. Next generation sequencing of the patient tumor.

\begin{tabular}{llllc}
\hline Gene & AA Change & Type & Allele call & Abundance \\
\hline BRCA2 & N372H & SNP & Homozygous & \\
BRIP1 & R439X & SNP & Homozygous & $48 \%$ \\
CYP2D6 & P34S & SNP & Homozygous & \\
CYP3A5 & CYP3A5 3 & SNP & Homozygous & \\
EGFR & R521K & SNP & Homozygous & \\
ERBB2 & I655V & SNP & Homozygous & \\
ERBB2 & P1170A & SNP & Heterozygous & \\
GSTM1 & & Deletion & Homozygous & \\
GSTT1 & & Deletion & Homozygous & \\
KRAS & G12D & SNP & & $5 \%$ \\
NQO1 & P187S & SNP & Homozygous & \\
PTEN & R173C & SNP & & $37 \%$ \\
UGT1A1 & 6/7TA & SNP & Heterozygous & \\
\hline
\end{tabular}

was according to the Principles of Laboratory Animal Care (NIH \#85-23, 1985 version). All animal studies were according to the Institutional Animal Care and Use Committee of Zhejiang University, and the approval ID was SYXK (ZHE) 2005-0072.

Multiple gene mutation analysis by next generation sequencing. The sequencing including 416 gene exons was conducted by Geneseeq Technology Inc. (Nanjing, China). ctDNA was extracted from patient's tumor. The purified ctDNA is quantified by a Picogreen fluorescence assay using the provided lambda DNA standards (Invitrogen Life Technologies, Carlsbad, CA, USA). Then, library construction with the KAPA Hyper DNA Library Prep Kit, containing mixes for end repair, dA addition and ligation, were performed in 96-well plates (Eppendorf). Dual-indexed sequencing libraries are PCR amplified for 4-7 cycles. The 5'-biotinylated probe solution is provided as capture probes, the baits target 416 cancer-related genes. $1 \mu \mathrm{g}$ of each ctDNA-fragment sequencing library is mixed with $5 \mu \mathrm{g}$ of human Cot-1 DNA, $5 \mu \mathrm{g}$ of salmon sperm DNA, and 1 unit adaptor-specific blocker DNA in hybridization buffer, heated for $10 \mathrm{~min}$ at $95^{\circ} \mathrm{C}$, and held for $5 \mathrm{~min}$ at $65^{\circ} \mathrm{C}$ in the thermocycler. Within $5 \mathrm{~min}$, the capture probes are added to the mixture, and the solution hybridization is performed for $16-18 \mathrm{~h}$ at $65^{\circ} \mathrm{C}$. After hybridization is complete, the captured targets are selected by pulling down the biotinylated probe/target hybrids using streptavidin-coated magnetic beads, and off-target library is removed by washing with wash buffer. The PCR master mix is added to directly amplify (6-8 cycles) the captured library from the washed beads. After amplification, the samples are purified by AMPure XP beads, quantified by qPCR (Kapa Biosystems, Inc., Wilmington, MA, USA) and sized on bioanalyzer 2100 (Agilent Technologies, Inc., Santa Clara, CA, USA). Libraries are normalized to $2.5 \mathrm{nM}$ and pooled. Deep Sequencing is performed on Illumina HiSeq 4000 using PE75 V1 kit. Cluster generation and sequencing is performed according to manufacturer's protocol. Base calling was performed using bcl2fastq v2.16.0.10 (Illumina, Inc., San Diego, CA, USA) to generate sequence reads in FASTQ format (Illumina 1.8+ encoding). 


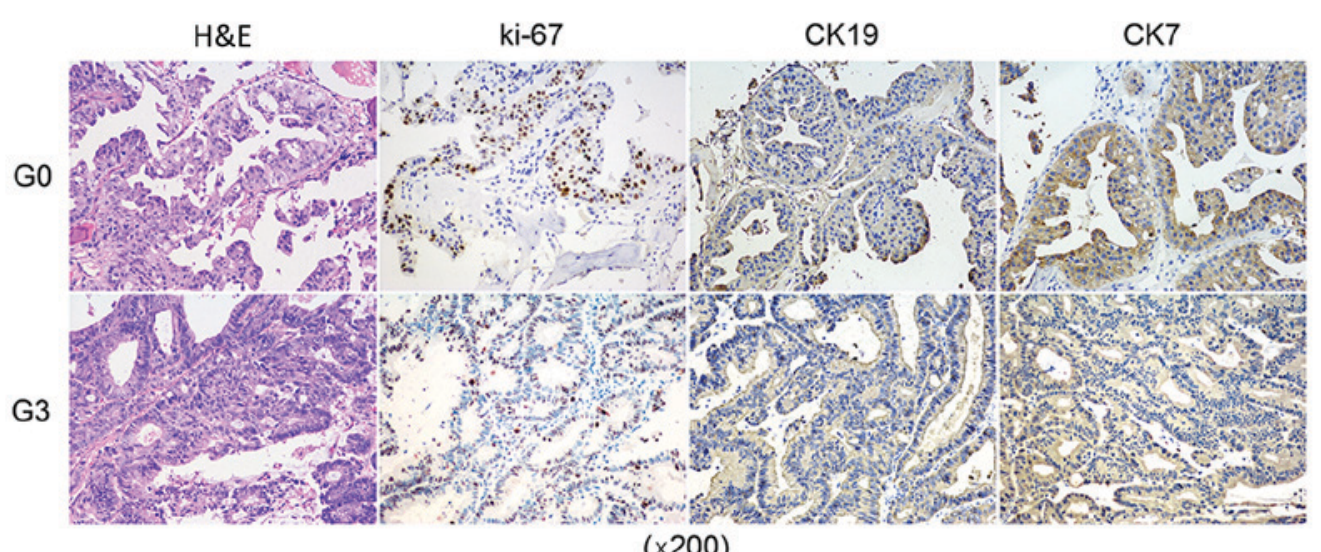

$(x 200)$

Figure 1. Immunohistochemical expressions compaired with PDX and patient tumor. The pathological characteristics of the third passage PDX xenograft was in accordance with the original patient sample. PDX, patient-derived xenograft; H\&E, hematoxylin and eosin.

Quality control (QC) was applied with Trimmomatic (27). High quality reads were mapped to the human genome (hg19, GRCh37 Genome Reference Consortium Human Reference 37) using modified BWA aligner 0.7.12 (28) with BWA-MEM algorithm and default parameters to create SAM files. Picard 1.119 (http://picard.sourceforge.net/) was used to convert SAM files to compressed BAM files which were then sorted according to chromosome coordinates. The Genome Analysis Toolkit (29) (GATK, version 3.4-0) was modified and used to locally realign the BAMs files at intervals with indel mismatches and recalibrate base quality scores of reads in BAM files (30). Single nucleotide variants (SNVs) and short insertions/deletions (indels) were identified using VarScan2 2.3.9 (31) with minimum variant allele frequency threshold set at 0.01 and P-value threshold for calling variants set at 0.05 to generate Variant Call Format (VCF) files. All SNVs/indels were annotated with ANNOVAR, and each SNV/indel was manually checked with the Integrative Genomics Viewer (32) (IGV). Copy number variations (CNVs) were identified using ADTEx 1.0.4 (33). The 416 gene exons sequencing report from Geneseeq Technology Inc also provided the drug treatment suggestions.

Treatment protocol. From the 3rd generation, PDX tumors were permitted to grow to a volume of $150-200 \mathrm{~mm}^{3}$, then mice were randomized ( 6 mice with tumors per group and housed in per rearing cage) and dosing was administrated (AZD6244, 50 mg/kg p.o. qd; Capecitabine, $1.0 \mathrm{mM} / \mathrm{kg}$ p.o. qd) for 4 weeks. Mice were weighed for signs of toxicity and tumor size was evaluated once per week. TGI (Relative tumor growth inhibition) was calculated using the following formula: (1-T/C)\%, where $\mathrm{T}$ means the relative tumor volume of the treated mice, and $\mathrm{C}$ means the relative tumor volume of the control mice.

Immunohistochemistry. Specimen were fixed by 10 neutral formalin, then embedded in paraffin, sectioned ( $5 \mu \mathrm{m}$ thick) and placed on slides for marker analysis. Sections were incubated with the primary antibodies overnight at $4^{\circ} \mathrm{C}$, after blocking nonspecific antibody bindings. The streptavidin-biotin peroxidase complex method (Lab Vision, Nairobi, Kenya) was used for immunohistochemistry. The slides were photographed using an Olympus BX60 (Olympus, Hamburg, Germany).
Statistical analysis. Results were presented as mean \pm SD. Calculation and statistics were performed with Excel 2010 (Microsoft Corporation, Redmond, WA, USA) and GraphPad Prism 5 (GraphPad Software Inc., La Jolla, CA, USA). One-way ANOVA were used to analyze the significance of differences among groups. $\mathrm{P}<0.05$ was considered statistically significant.

\section{Results}

Patient characteristics and PDX model establishment. Pancreatic cancer bone metastasis (diagnosed as adenocarcinoma) tissues obtained at surgery from a 67-year-old female patient were subcutaneously implanted into BALB/c nude mice for the PDX model establishment. Tumors were re-implanted in new mice after reaching a volume of $1,000 \mathrm{~mm}^{3}$ as model passaging. The PDX model was serially passaged in animals 3 times. In order to further evaluate the PDX xenograft, immunohistochemical test was performed to identify if the patient's characteristics were retained in the PDX. Immunohistochemical expressions of CK19, CK7, and ki67 as well as the H\&E staining showed that the pathological characteristics of the third passage xenograft was in accordance with the original patient sample (Fig. 1).

Next generation sequencing for drug efficacy prediction. The sequencing of pancreatic cancer bone metastasis tissues of the patient tumor was conducted by Geneseeq Technology Inc. Totally, 13 pancreatic cancer-associated gene polymorphisms/mutations were found out of the 416 genes sequenced (Tables I and II). Based on the sequencing results and associated literatures, there were no under-clinical-trial targeted therapies of pancreatic cancer directly suitable for the genes detected. Therefore AZD6244 (AZD for short, also named as Selumetinib), a highly selective inhibitor against $M E K 1$, was chosen as a potential therapy whose antitumor efficacy would then be evaluated in our PDX model.

Efficacy evaluation of AZD6244 based on PDX model. To test whether the PDX model of pancreatic cancer bone metastasis was sensitive to the suggested therapy, antitumor-growth ability of AZD6244 were evaluated (Capecitabine for positive control). Since tumors volume reached $150-200 \mathrm{~mm}^{3}$, 
Table II. 416 genes for analysis.

\begin{tabular}{|c|c|c|c|c|c|c|c|}
\hline $\mathrm{ABCC} 2$ & DMNT3A & KDR & RAF1 & ABCC2 & DMNT3A & KDR & RAF1 \\
\hline ACTB & DNM2 & KIF1B & RARA & CD58 & GADD45B & NRAS & SUZ12 \\
\hline ADH1B & DOCK1 & KIT & RASGEF1A & CD70 & GATA1 & NRG1 & SYN3 \\
\hline AIP & DOT1L & КМТ2В & RB1 & CDA & GATA2 & NSD1 & TCN2 \\
\hline AKT1 & DPYD & KMT2C & RECQL4 & CDC73 & GATA3 & NT5C2 & TEK \\
\hline AKT2 & DUSP2 & KRAS & RELN & CDH1 & GATA4 & NTRK1 & TEKT4 \\
\hline AKT3 & EBF1 & LEF1 & RET & CDK10 & GATA6 & PAG1 & TERC \\
\hline ALDH2 & ECT2L & LMO1 & RHBDF2 & CDK12 & GNA11 & PAK3 & TERT \\
\hline ALK & EED & LSP1 & RHOA & CDK4 & GNA13 & PALB2 & TET2 \\
\hline AMER1 & EGFR & LYN & RICTOR & CDK6 & GNAQ & PARK2 & TGFBR2 \\
\hline AP3B1 & EGR1 & LYST & RNF146 & CDK8 & GNAS & PAX5 & TLE1 \\
\hline APC & EP300 & LZTR1 & RNF43 & CDKN1B & GPC3 & PBRM1 & TLE4 \\
\hline AR & EPCAM & MAP2K1 & ROS1 & CDKN1C & GRIN2A & $\mathrm{PC}$ & TMEM127 \\
\hline ARAF & EPHA3 & MAP2K2 & ROS1 & CDKN2A & GRM3 & PDCD1 & TMPRSS2 \\
\hline ARID1A & ERBB2 & MAP2K4 & RPTOR & CDKN2B & GSTM1 & PDCD1LG2 & TNFAIP3 \\
\hline ARID2 & ERBB3 & MAP3K1 & RRM1 & CDKN2C & GSTP1 & PDGFRA & TNFRSF14 \\
\hline ARID5B & ERBB4 & MCL1 & RUNX1 & CEBPA & GSTT1 & PDGFRB & TNFRSF17 \\
\hline ASXL1 & ERCC1 & MDM2 & SBDS & CEP57 & HBA1 & PDK1 & TNFRSF19 \\
\hline ATM & ERCC2 & MDM4 & SDHA & CHD4 & HBA2 & PHF6 & TOP1 \\
\hline ATR & ERCC3 & MECOM & SDHAF2 & CHEK1 & HBB & PHOX2B & TOP2A \\
\hline ATRX & ERCC4 & MED12 & SDHB & CHEK2 & HDAC1 & PICK3R1 & TP53 \\
\hline AURKA & ERCC5 & MEF2B & SDHC & CKS1B & HDAC2 & PIK3C3 & ТР63 \\
\hline AURKB & ESR1 & MEN1 & $\begin{array}{l}\text { SDHD } \\
\text { SERP? }\end{array}$ & CREBBP & HDAC4 & PIK3CA & TPMT \\
\hline AXIN1 & ETV1 & MET & $\begin{array}{l}\text { SERP2 } \\
\text { SETRP1 }\end{array}$ & CRKL & HDAC7 & PIK3CD & TRAF2 \\
\hline AXL & ETV4 & MGMT & SETBP1 & CROT & HGF & PIK3R1 & TRAF3 \\
\hline B2M & EWSR1 & MITF & $\begin{array}{l}\text { SETD2 } \\
\text { SE3B1 }\end{array}$ & CSF1R & HNF1A & PIK3R2 & TRAF5 \\
\hline BAP1 & $\begin{array}{l}\text { EXT1 } \\
\text { EXT2 }\end{array}$ & MLH1 & $\begin{array}{l}\text { SF3B1 } \\
\text { SGK1 }\end{array}$ & CSF3R & HNF1B & PLCE1 & TSC1 \\
\hline $\begin{array}{l}\text { BARDI } \\
\text { BAT3 }\end{array}$ & $\begin{array}{l}\text { EX12 } \\
\text { EZH2 }\end{array}$ & $\begin{array}{l}\text { MLL } \\
\text { MLLT10 }\end{array}$ & $\begin{array}{l}\text { SHK1 } \\
\text { SH2D1A }\end{array}$ & CTCF & HRAS & PLK1 & TSC2 \\
\hline $\begin{array}{l}\text { BA13 } \\
\text { BCL2 }\end{array}$ & FANCA & MLPH & SLX4 & CTLA4 & ID3 & PMS1 & TSHR \\
\hline BCL2L1 & FANCB & MPL & SMAD2 & CTNNB1 & IDH1 & PMS2 & TTF1 \\
\hline BCL2L2 & FANCC & MRE11A & SMAD3 & CUX1 & IDH2 & POLD1 & TUBB3 \\
\hline BCORL1 & FANCD2 & MSH2 & SMAD4 & CXCR4 & IGF1R & POLD3 & TYMS \\
\hline BIM(BCL2L11) & FANCE & MSH3 & SMAD7 & CYLD & IGF2 & POLE & TYR \\
\hline BLM & FANCF & MSH6 & SMARCA4 & CYP2B6*6 & IKBKE & POT1 & U2AF1 \\
\hline BMPR1A & FANCG & MTHFR & SMARCB1 & CYP2B6*6 & IKZF1 & PPP2R1A & UGT1A1 \\
\hline BRAF & FANCI & MTOR & SMC1A & CYP2C $19 * 2$ & IKZF2 & PRDM1 & UNC13D \\
\hline BRCA1 & FANCL & MUTYH & SMC3 & CYP2C9*3 & IKZF3 & PRF1 & VEGFA \\
\hline BRCA2 & FANCM & MYC & SMO & CYP2D6 & IL13 & PRKAR1A & VHL \\
\hline BRD4 & FAT1 & MYCL1 & SOX2 & CYP2D6*3 & IL7R & PRKCI & WISP3 \\
\hline BRIP1 & FBXO11 & MYCN & SPOP & CYP2D6* 4 & INPP4B & PTCH1 & WRN \\
\hline BTG2 & FCGR2B & MYD88 & $\mathrm{SRC}$ & CYP2D6* 6 & INPP5D & PTEN & WT1 \\
\hline BTK & FGF19 & MYNN & SRSF2 & CYP3A4*4 & IRF1 & PTPN11 & XIAP \\
\hline BTLA & FGFR1 & NBN & STAG2 & CYP3A5*3 & IRF2 & PTPN2 & XPA \\
\hline BUB1B & FGFR2 & NCSTN & STAT3 & DAB2 & IRF4 & PTPN6 & XPC \\
\hline c11orf30 & FGFR3 & NF1 & STAT5A & DAXX & IRF8 & PTPRO & XPO1 \\
\hline CALR & FGFR4 & NF2 & STAT5B & DDB2 & JAK 1 & QKI & XRCC1 \\
\hline CBL & $\mathrm{FH}$ & NFKBIA & STIL & DDR2 & JAK2 & RAC1 & YAP1 \\
\hline CCND1 & FIP1L1 & NKX2-1 & STK11 & DDX1 & JAK3 & RAD21 & ZAP70 \\
\hline CCNE1 & FLCN & NOTCH1 & STMN1 & DHFR & JARID2 & RAD50 & ZBTB20 \\
\hline ССТ6В & FLT1 & NOTCH2 & STX11 & DICER1 & JUN & RAD51 & ZNF217 \\
\hline CD22 & FLT3 & NPM1 & STXBP2 & DIS3L2 & KDM2B & RAD51C & ZNF703 \\
\hline CD274 & FLT4 & NQO1 & SUFU & DLG2 & KDM5A & RAD51D & ZRSR2 \\
\hline
\end{tabular}

Table II. Continued. 
A

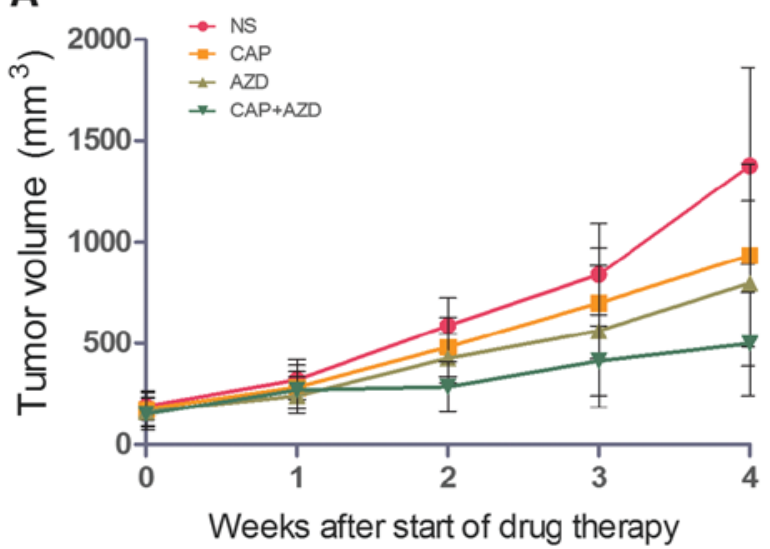

B

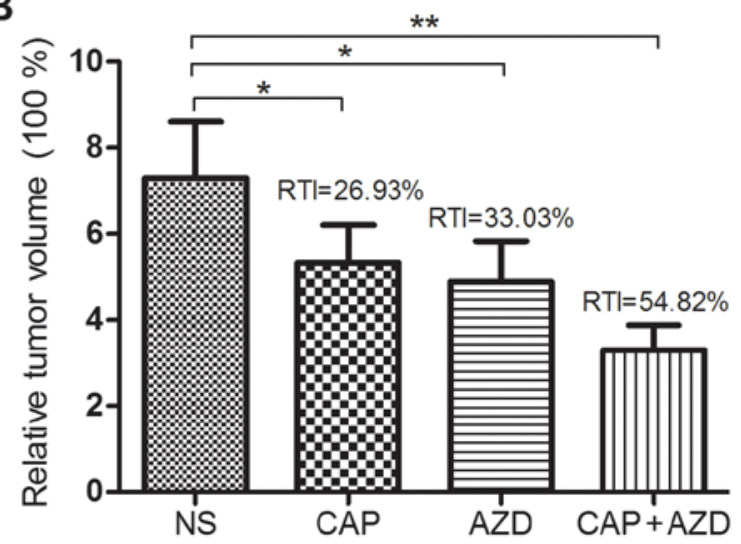

Figure 2. (A) Antitumor-growth ability of AZD6244. (B) The single AZD6244 exhibited better efficacy than Capecitabine, while the combination of both shown a significant synergistic effect.

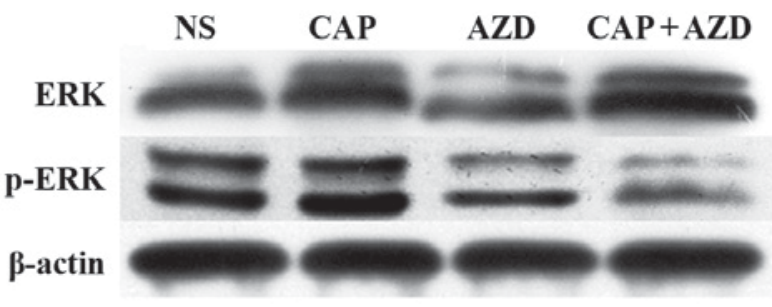

Figure 3. Western blot analysis for changes of ERK and p-ERK expressions in all groups. The p-ERK expressions were significantly suppressed in both single and combined AZD6244 groups. ${ }^{* * *} \mathrm{P}<0.01,{ }^{*} \mathrm{P}<0.05$.
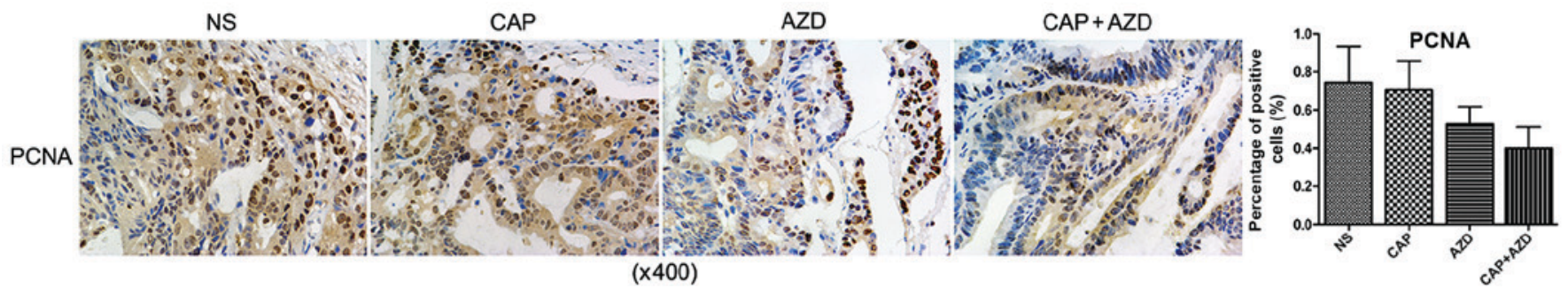

Figure 4. Immunohistochemical staining shown that PCNA expressions in the AZD6244-treated groups were significantly suppressed.
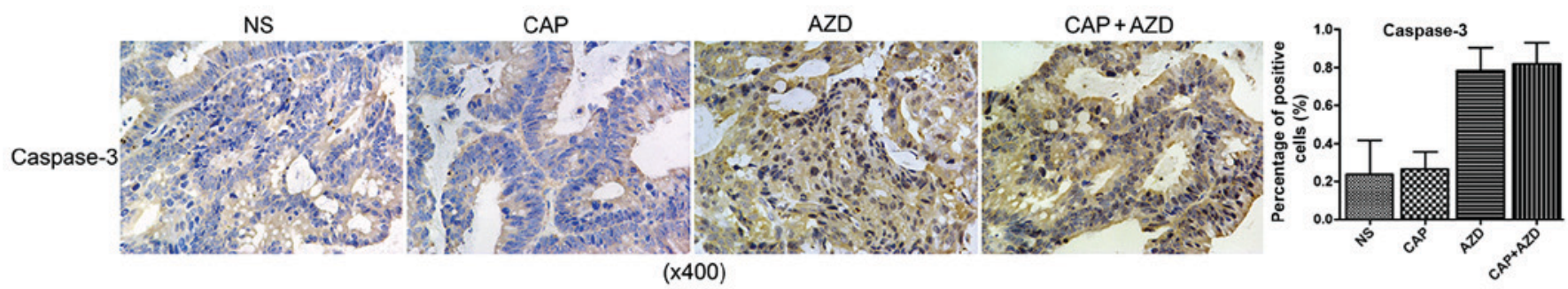

Figure 5. Immunohistochemical staining shown that caspase-3 expressions in the AZD6244-treated groups were significantly upregulated.

orally administration of AZD6244 $(50 \mathrm{mg} / \mathrm{kg})$, Capecitabine $(1.0 \mathrm{mM} / \mathrm{kg})$ or saline were then given once a day for 28 days. The mice were killed and excised tumors were measured. Then, relative tumor growth inhibition (TGI) was calculated as per the following formula: $(1-\mathrm{T} / \mathrm{C}) \%$, where $\mathrm{T}$ is relative tumor volume of treated group mice, and $\mathrm{C}$ is relative tumor volume of control group mice. We found that single AZD6244 exhibited better efficacy (TGI, 33.03\%) than Capecitabine (TGI, 26.93\%), although without statistical significance. While the combination of both shown a significant synergistic effect, with TGI of $54.82 \%$ (Fig. 2). By western blotting, we evaluated the changes of ERK and p-ERK expressions in all groups, to 
find that p-ERK expressions were significantly suppressed in both single and combined AZD6244 groups (Fig. 3). By immunohistochemical staining, we found that PCNA (proliferating cell nuclear antigen) expressions in the AZD6244-treated groups were significantly suppressed, while caspase-3 (one of apoptosis associated antigens) expressions were significantly upregulated (Figs. 4 and 5). Therefore, AZD6244 was evaluated effective for the pancreatic cancer PDX model, thus might provide potential efficay in the clinical treatment of the very pancreatic cancer patient.

\section{Disscussion}

Novel technologies contribute to the progress of the drug screening of pancreatic cancer during recent years. PDX models are being used for pancreatic cancer research in a series of studies $(2,7,34,35)$, while NGS technologies contribute to the translational research of pancreatic cancer (36-38). Multiple clinical studies have showed NGS and PDX will ameliorate personalized medicine and will be necessary for discovering novel therapeutic targets and biomarkers (39). With the progress of these technologies, both are getting economically availble for patients. In our study, we combined PDX and NGS as an promising pattern of individualized drug screening to improve the clinical treatment of pancreatic cancer patients.

The PDX model of pancreatic cancer bone metastasis we established was comfirmed as highly molecularly stable with clinical patients in our study. Immunohistochemical expressions of CK19, CK7, and ki67 as well as the H\&E staining showed that the pathological characteristics of the third passage xenograft was in accordance with the original patient tumor. Therefore, our PDX model could be considered as an 'Avatar' or a 'stand-in' of our pancreatic cancer patient, which would be a quite promising platform for drug screeing and evaluation.

In order to select the potential therapies customized for the pancreatic cancer patient, the bone metastasis tumor tissues were used for NGS detection (Geneseeq Technology Inc). However, based on the sequencing results and associated literatures, we found no under-clinical-trial targeted therapies of pancreatic cancer directly suitable for the genes detected. The sequencing report from Geneseeq Technology Inc also provided the alternative drug treatment suggestions, and MEK1 inhibitor was one of the most promising targeted therapies suggested. Then we concentrated on $M E K 1$, a downstream gene of $K R A S$, which might be a potential target for treatment. Therefore we chose AZD6244, a MEK1 inhibitor, as a potential therapy which would then be evaluated in our PDX model.

In our study, we found that single AZD6244 exhibited better efficacy than Capecitabine, although without statistical significance. While the combination of both shown a significant synergistic effect, with TGI of 54.82\%. AZD6244 significantly suppressed p-ERK expressions of the pancreatic cancer PDX model. AZD6244 significantly suppressed tumor cell proliferation and upregulated tumor cell apoptosis. Several studies have evaluated the effect of AZD6244 in pancreatic cancer in preclinical and clinical phase, and AZD6244 was shown to be effective in combination with EGFR/PIK3CA/STAT3 inhibitors in patients with pancreatic cancer (40-42). While we have shown that AZD6244 also has a synergistic effect in combination with Capecitabine. In addition, it was suggested that AZD6244 alone was mainly cytostatic, and apoptosis was mainly induced by combination therapies targeting multiple pathways (43). While here in the present study, we shown that AZD6244 also suppressed tumor cell proliferation as a sinlge agent. Therefore, AZD6244 was evaluated as effective for the pancreatic cancer PDX model, thus might provide potential efficay in the clinical treatment of this pancreatic cancer patient.

In our study, AZD6244, a highly selective MEK1 inhibitor, was evaluated as effective for the pancreatic cancer PDX model, and thus might provide potential efficay in the clinical treatment of this pancreatic cancer patient. Although only one targeted agent was evaluated, we have successfully shown PDX-NGS based drug screening as a novel promising pattern of individualized drug screening to improve the clinical treatment of pancreatic cancer patients.

\section{Acknowledgements}

The present study was supported by the National Natural Science Foundation of China (grant no. 81374014), Zhejiang Provincial Science and Technology Projects (grant nos. 2015C33264, 2017C33212 and 2017C33213), and Zhejiang Provincial Medical and Healthy Science and Technology Projects (grant nos. 2013KYA228 and 2016KYA180).

\section{References}

1. Oettle H, Post S, Neuhaus P, Gellert K, Langrehr J, Ridwelski K, Schramm H, Fahlke J, Zuelke C, Burkart C, et al: Adjuvant chemotherapy with gemcitabine vs observation in patients undergoing curative-intent resection of pancreatic cancer: A randomized controlled trial. JAMA 297: 267-277, 2007.

2. Huang L, Holtzinger A, Jagan I, BeGora M, Lohse I, Ngai N, Nostro C, Wang R, Muthuswamy LB, Crawford HC, et al: Ductal pancreatic cancer modeling and drug screening using human pluripotent stem cell- and patient-derived tumor organoids. Nat Med 21: 1364-1371, 2015.

3. Mattie M, Christensen A, Chang MS, Yeh W, Said S, Shostak Y, Capo L, Verlinsky A, An Z, Joseph I, et al: Molecular characterization of patient-derived human pancreatic tumor xenograft models for preclinical and translational development of cancer therapeutics. Neoplasia 15: 1138-1150, 2013.

4. Vincent A, Herman J, Schulick R, Hruban RH and Goggins M: Pancreatic cancer. Lancet 378: 607-620, 2011.

5. Sohal DP, Mangu PB, Khorana AA, Shah MA, Philip PA, O'Reilly EM, Uronis HE, Ramanathan RK, Crane CH, Engebretson A, et al: Metastatic pancreatic cancer: American society of clinical oncology clinical practice guideline. J Clin Oncol 34: 2784-2796, 2016.

6. Garrido-Laguna I and Hidalgo M: Pancreatic cancer: from state-of-the-art treatments to promising novel therapies. Nat Rev Clin Oncol 12: 319-334, 2015.

7. Boj SF, Hwang CI, Baker LA, Chio II, Engle DD, Corbo V, Jager M, Ponz-Sarvise M, Tiriac H, Spector MS, et al: Organoid models of human and mouse ductal pancreatic cancer. Cell 160: 324-338, 2015

8. Heestand GM and Kurzrock R: Molecular landscape of pancreatic cancer: Implications for current clinical trials. Oncotarget 6: 4553-4561, 2015.

9. The Lancet Oncology: Pancreatic cancer: Cause for optimism? Lancet Oncol 17: 845, 2016.

10. Iguchi H, Yasuda M, Matsuo T, Sumii T and Funakoshi A: Clinical features and management of pancreatic cancer with bone metastases. Nihon Shokakibyo Gakkai Zasshi 101: 872-878, 2004 (In Japanese).

11. Pneumaticos SG, Savidou C, Korres DS and Chatziioannou SN: Pancreatic cancer's initial presentation: Back pain due to osteoblastic bone metastasis. Eur J Cancer Care (Engl) 19: 137-140, 2010. 
12. Hidalgo M, Bruckheimer E, Rajeshkumar NV, Garrido-Laguna I, De Oliveira E, Rubio-Viqueira B, Strawn S, Wick MJ, Martell J and Sidransky D: A pilot clinical study of treatment guided by personalized tumorgrafts in patients with advanced cancer. Mol Cancer Ther 10: 1311-1316, 2011.

13. Johnson JI, Decker S, Zaharevitz D, Rubinstein LV, Venditti JM, Schepartz S, Kalyandrug S, Christian M, Arbuck S, Hollingshead M and Sausville EA: Relationships between drug activity in NCI preclinical in vitro and in vivo models and early clinical trials. Br J Cancer 84: 1424-1431, 2001.

14. Aparicio S, Hidalgo $M$ and Kung AL: Examining the utility of patientderived xenograft mouse models. Nat Rev Cancer 15: 311-316, 2015

15. Stratton MR, Campbell PJ and Futreal PA: The cancer genome. Nature 458: 719-724, 2009.

16. Hanahan D and Weinberg RA: Hallmarks of cancer: The next generation. Cell 144: 646-674, 2011.

17. Chakradhar S: Colorectal cancer: 5 big questions. Nature 521 S16, 2015.

18. Hamilton SR: Molecular pathology. Mol Oncol 6: 177-181, 2012

19. Verweij J, de Jonge M, Eskens F and Sleijfer S: Moving molecular targeted drug therapy towards personalized medicine: Issues related to clinical trial design. Mol Oncol 6: 196-203, 2012.

20. Garay JP and Gray JW: Omics and therapy-A basis for precision medicine. Mol Oncol 6: 128-139, 2012.

21. Woollard PM, Mehta NA, Vamathevan JJ, Van Horn S, Bonde BK and Dow DJ: The application of next-generation sequencing technologies to drug discovery and development. Drug Discov Today 16: 512-519, 2011.

22. Macconaill LE and Garraway LA: Clinical implications of the cancer genome. J Clin Oncol 28: 5219-5228, 2010.

23. Belchis DA, Tseng LH, Gniadek T, Haley L, Lokhandwala P, Illei P Gocke CD, Forde P, Brahmer J, Askin FB, et al: Heterogeneity of resistance mutations detectable by next-generation sequencing in TKI-treated lung adenocarcinoma. Oncotarget 7: 45237-45248, 2016.

24. Jee J, Rasouly A, Shamovsky I, Akivis Y, Steinman SR, Mishra B and Nudler E: Rates and mechanisms of bacterial mutagenesis from maximum-depth sequencing. Nature 534: 693-696, 2016.

25. Jin K, He K, Han N, Li G, Wang H, Xu Z, Jiang H, Zhang J and Teng L: Establishment of a PDTT xenograft model of gastric carcinoma and its application in personalized therapeutic regimen selection. Hepatogastroenterology 58: 1814-1822, 2011.

26. Zhonghai Guan, Xiangheng Chen, Xiaoxia Jiang, Zhongqi Li, Xiongfei Yu, Ketao Jin, Jiang Cao and Lisong Teng: Establishing a patient-derived colorectal cancer xenograft model for translational research. Int J Clin Exp Med 9: 21346-21357, 2016.

27. Bolger AM, Lohse M and Usadel B: Trimmomatic: A flexible trimmer for Illumina sequence data. Bioinformatics 30: 2114-2120, 2014.

28. Li H and Durbin R: Fast and accurate short read alignment with Burrows-Wheeler transform. Bioinformatics 25: 1754-1760, 2009.

29. McKenna A, Hanna M, Banks E, Sivachenko A, Cibulskis K, Kernytsky A, Garimella K, Altshuler D, Gabriel S, Daly M and DePristo MA: The genome analysis toolkit: A mapreduce framework for analyzing next-generation DNA sequencing data Genome Res 20: 1297-1303, 2010.

30. Van der Auwera GA, Carneiro MO, Hartl C, Poplin R, Del Angel G, Levy-Moonshine A, Jordan T, Shakir K, Roazen D, Thibault J, et al: From FastQ data to high confidence variant calls: The Genome Analysis Toolkit best practices pipeline. Curr Protoc Bioinformatics 43: 1-33, 2013.
31. Koboldt DC, Zhang Q, Larson DE, Shen D, McLellan MD, Lin L, Miller CA, Mardis ER, Ding L and Wilson RK: VarScan 2: Somatic mutation and copy number alteration discovery in cancer by exome sequencing. Genome Res 22: 568-576, 2012

32. Robinson JT, Thorvaldsdóttir H, Winckler W, Guttman M, Lander ES, Getz G and Mesirov JP: Integrative genomics viewer. Nat Biotechnol 29: 24-26, 2011.

33. Amarasinghe KC, Li J, Hunter SM, Ryland GL, Cowin PA, Campbell IG and Halgamuge SK: Inferring copy number and genotype in tumour exome data. BMC Genomics 15: 732, 2014.

34. Lipner MB, Marayati R, Deng Y, Wang X, Raftery L, O'Neil BH and Yeh JJ: Metformin treatment does not inhibit growth of pancreatic cancer patient-derived xenografts. PLoS One 11: e0147113, 2016.

35. Walters DM, Stokes JB, Adair SJ, Stelow EB, Borgman CA, Lowrey BT, Xin W, Blais EM, Lee JK, Papin JA, et al: Clinical, molecular and genetic validation of a murine orthotopic xenograft model of pancreatic adenocarcinoma using fresh human specimens. PLoS One 8: e77065, 2013.

36. Jones S, Hruban RH, Kamiyama M, Borges M, Zhang X, Parsons DW, Lin JC, Palmisano E, Brune K, Jaffee EM, et al: Exomic sequencing identifies PALB2 as a pancreatic cancer susceptibility gene. Science 324: 217, 2009.

37. Liang WS, Craig DW, Carpten J, Borad MJ, Demeure MJ, Weiss GJ, Izatt T, Sinari S, Christoforides A, Aldrich J, et al: Genome-wide characterization of pancreatic adenocarcinoma patients using next generation sequencing. PLoS One 7: e43192, 2012.

38. Biankin AV, Waddell N, Kassahn KS, Gingras MC, Muthuswamy LB, Johns AL, Miller DK, Wilson PJ, Patch AM, Wu J, et al: Pancreatic cancer genomes reveal aberrations in axon guidance pathway genes. Nature 491: 399-405, 2012.

39. Garralda E, Paz K, López-Casas PP, Jones S, Katz A, Kann LM, López-Rios F, Sarno F, Al-Shahrour F, Vasquez D, et al: Integrated next-generation sequencing and avatar mouse models for personalized cancer treatment. Clin Cancer Res 20: 2476-2484, 2014

40. Ko AH, Bekaii-Saab T, Van Ziffle J, Mirzoeva OM, Joseph NM, Talasaz A, Kuhn P, Tempero MA, Collisson EA, Kelley RK, et al: A multicenter, open-label phase II clinical trial of combined MEK plus EGFR inhibition for chemotherapy-refractory advanced pancreatic adenocarcinoma. Clin Cancer Res 22: 61-68, 2016.

41. Diep CH, Munoz RM, Choudhary A, Von Hoff DD and Han H: Synergistic effect between erlotinib and MEK inhibitors in KRAS wild-type human pancreatic cancer cells. Clin Cancer Res 17: 2744-2756, 2011.

42. Zhao C, Xiao H, Wu X, Li C, Liang G, Yang S and Lin J: Rational combination of MEK inhibitor and the STAT3 pathway modulator for the therapy in K-Ras mutated pancreatic and colon cancer cells. Oncotarget 6: 14472-1487, 2015 .

43. Alagesan B, Contino G, Guimaraes AR, Corcoran RB, Deshpande V, Wojtkiewicz GR, Hezel AF, Wong KK, Loda M, Weissleder R, et al: Combined MEK and PI3 K inhibition in a mouse model of pancreatic cancer. Clin Cancer Res 21: 396-404, 2015 . 